\title{
First principle study of silver nanoparticle interactions with antimalarial drugs extracted from Artemisia annua plant
}

\author{
Mahmood Akbari (D) Razieh Morad • Malik Maaza
}

Received: 4 August 2020 / Accepted: 19 October 2020 /Published online: 27 October 2020

(C) Springer Nature B.V. 2020

\begin{abstract}
Silver nanoparticles have a great potential in a broad range of applications such as drug-delivery carriers because of their antiviral and antibacterial properties. In this study, the coating properties of silver nanoparticle (size range of $1.6 \mathrm{~nm}$ ) with three common anti-malarial drugs, Artemisinin, Artemether, and Artesunate have been studied by using the quantum mechanical and classical atomistic molecular dynamics simulation in order to use as the drug delivery to treat malaria and COVID-19 diseases. The optimized structure, frequencies, charge distribution, and the electrostatic potential maps of the three drug molecules were simulated by using the density functional theory (DFT) at the B3LYP/6-311++g(d,p) level of theory. Then, molecular dynamics simulation was used to study the coating of AgNP with each of these drugs. The affinity of interaction was obtained as Artesunate $>$ Artemether
\end{abstract}

This article is part of the topical collection: Nanotechnology Convergence in Africa

Guest Editors: Mamadou Diallo, Abdessattar Abdelkefi, and Bhekie Mamba

M. Akbari $(\bowtie) \cdot$ R. Morad $\cdot$ M. Maaza

UNESCO-UNISA Africa Chair in Nanoscience \&

Nanotechnology (U2ACN2), College of Graduate Studies, University of South Africa (UNISA), Pretoria, South Africa e-mail: makbari@tlabs.ac.za

M. Akbari · R. Morad • M. Maaza

Nanoscience African Network (NANOAFNET), Material

Research Division, iThemba LABS-National Research

Foundation, Somerset West 7129, South Africa
$>$ Artemisinin which is in agreement with the DFT results on the adsorption of drugs on the $\operatorname{Ag}(111)$ slab.

Keywords Silver nanoparticle - Artemisia annua plant . Artemisinin - Artemether - Artesunate - Density functional theory $\cdot$ Molecular dynamics simulations . Drug delivery

\section{Introduction}

For millennia, herbal folk medicines in Asia, Africa, and South America have been used to treat infectious diseases (Stojanoski 1999; Petrovska 2012). Amongst them, the Artemisia annua consisting of a group of plants known as wormwoods have been used for some medicinal purposes, including malaria, for centuries (Dalrymple 2013). Artemisinin, which is an important bioactive component found in Artemisia annua leaves and flowers, is extracted from this plant and is the basis for the WHO-recommended anti-malaria combination therapies used in millions of adults and children each year with few, if any, side effects (Wong et al. 2020). Artesunate and Artemether are the two most significant Artemisinin derivatives and promising novel drugs to treat pulmonary fibrosis by inhibiting pro-fibrotic molecules associated with pulmonary fibrosis (Wang et al. 2015; Suputtamongkol et al. 2001). Artemisinin and its derivatives exhibit significant properties such as a highantioxidant activity (due to its high phenolic content) (Ferreira et al. 2010) and contains sterols that show virus inhibitory potential (Abid Ali Khan et al. 1991). The 
most interesting intension in testing the antioxidant and antifibrotic effects of Artemisinin and its derivatives is considering their key role in lung fibrosis (Wang et al. 2015). The choice of which oral Artemisia annua's extract to use in different clinical situations has been largely empirical. Furthermore, this plant has a history of being safe, cheap, and widely available for therapies, although final technical analysis is demanded. Besides malaria, Artemisinin and its derivatives have efficiency against some viral and parasitic diseases, i.e., hepatitis B, schistosomiasis, Chagas disease, African sleeping sickness, and treatment of some cancers (Duffy and Mutabingwa 2006; White 2008; Efferth 2007). In addition, recently, some research groups at the Max Planck Institute of Colloids and Interfaces in Germany (Gilmore et al. 2020) and the University of Swat in Pakistan (Ul Haq et al. 2020) have shown in laboratory studies that aqueous and ethanolic extracts of specially bred sweet wormwood plants (Artemisia annua) are active against the new coronavirus that has caused the COVID-19 pandemic. These studies motivate scientists again to pay attention to this miracle traditional medicinal plant.

Drug delivery has long been a concern in nanomedicine (Patra et al. 2018; Ivanova et al. 2018). It has been shown that the best therapeutic effect can be achieved by loading the drug onto the nanocarriers such as metal nanoparticles that can accumulate near the target cells. This method can enhance permeability and retention effect of the drug (Bazban-shotorbani et al. 2015). Metal nanoparticles, such as silver and gold with the size of 1-100 nm, possess different chemical, physical, and optical properties compared with those of their bulk structures while due to relatively high surface areas could exhibit various properties and applications in diverse fields (Aderibigbe 2017). In particular, silver nanoparticles (AgNPs) have attracted impressive attention toward the biomedicine-related assessment as unconventional antimicrobial agents (Alexander 2009; Nedelcu et al. 2014; Burdușel et al. 2018). Conjugation of drugs onto silver nanoparticles can protect them against the body's immune system, thereby extending their blood circulation time (Mody et al. 2010). The unique properties of AgNPs make these nanoparticles a potential therapeutic for the treatment of infectious diseases (Mody et al. 2010). The nanoparticles with smaller sizes show more stability and can interact with biomolecules both at the surface and inside cells (Rezaee et al. 2020). Silver nanoparticles engineered for biomedical applications must meet a series of conditions, such as being stable and not aggregate, biocompatible, selective to target cells or tissues, nontoxic, and affordable (Mody et al. 2010; Choi et al. 2008; Feng et al. 2000). Recently, the antiviral and immunomodulatory properties of silver nanoparticles have been studied extensively (Geraldo et al. 2016; Chowdhury et al. 2016; Tavaf et al. 2017; Henke et al. 2016; Galdiero et al. 2011; Villeret et al. 2018). For instance, Prusty and Swain synthesized a polyacrylamide/dextran nano-hydrogels hybrid system with covalently attached silver nanoparticles for the release of ornidazole (Prusty and Swain 2018). Also Morris demonstrated in vivo antiviral activity of AgNPs during respiratory syncytial virus (RSV) infection (Morris et al. 2019).

In the context of computational materials science, first principle Density Functional Theory (DFT) calculations allow the prediction and calculation of material behavior on the basis of quantum mechanical considerations, without requiring higher-order parameters such as fundamental material properties (Pakiari and Jamshidi 2007; Granatier et al. 2007; Aliakbari Tehrani et al. 2012). Alongside DFT, molecular dynamics (MD) simulation is a powerful computational method that can analyze the interactions between species in a system in atomistic level and reveals problems that are not observable experimentally (Antuek et al. 2003; Sambasivam et al. 2016). However, a recourse to MD simulation was needed to evaluate the interaction of drug and AgNP to predict the affinity sites of the drug to interact, which is a useful concept in designing effective combination for drug delivery purposes (Yousefpour et al. 2018; Kordzadeh et al. 2019).

In this study, the interactions of Artemisinin, Artemether, and Artesunate drugs with slab of sliver are investigated at the DFT level of theory, and the optimized geometrical and charge distributions are used as input for molecular dynamic simulation to find out the coating affinity of silver nanoparticle as the drug vehicle to efficient usage of these drugs and deliverer to the target agent.

\section{Computational methods}

The geometry optimization, frequencies, and electronic structure calculations including the charge distribution and the electrostatic potential maps of the three drug molecules were performed by using the density functional theory (DFT) at the B3LYP/6-311++g(d,p) level of theory 


\subsection{0}

\section{$-0.350$}
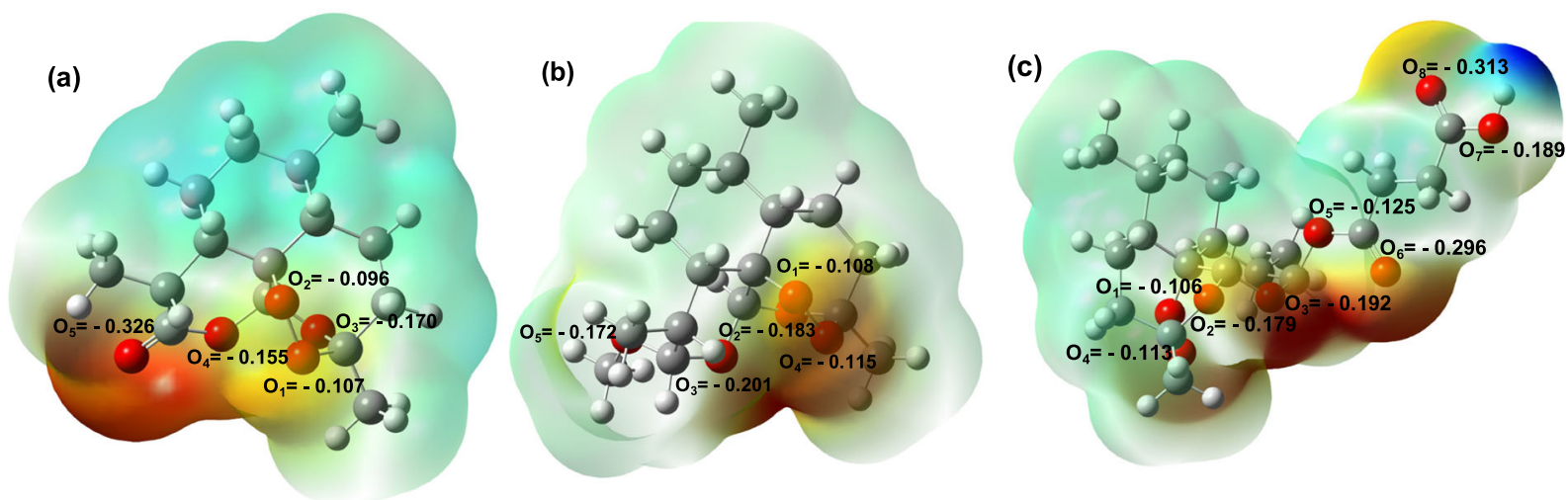

Fig. 1 Charge distribution (Hirshfeld point charges) and electrostatic potential map for three drug molecules Artemisinin (a), Artemether (b), and Artesunate (c). The geometry optimization of the drug molecules were carried out at the B3LYP/6-311++g(d,p) level of theory

with the Gaussian software package, version 09 (Frisch et al. 2009). Having no imaginary frequencies for optimized geometries confirms the true minima on the potential energy surfaces. The adsorption of the three drug molecules on a periodic slab of the $\operatorname{Ag}(111)$ surface was simulated within the generalized gradient approximation
(GGA) in the formulation of PBE (Perdew-BurkeErnzerhof) (Perdew et al. 1996) implemented in PWscf code of Quantum Espresso package (version 6.4.1) (Giannozzi et al. 2009). The D3-Grimme dispersion correction (Grimme et al. 2011) was considered and the electron-ion interaction was described by the ultra-soft
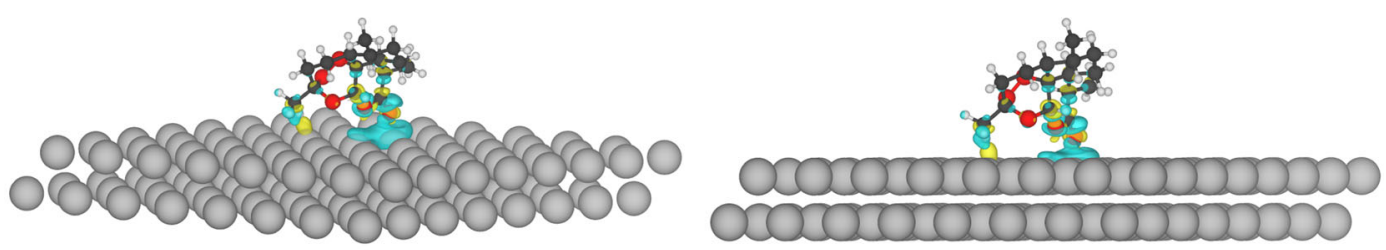

(a)
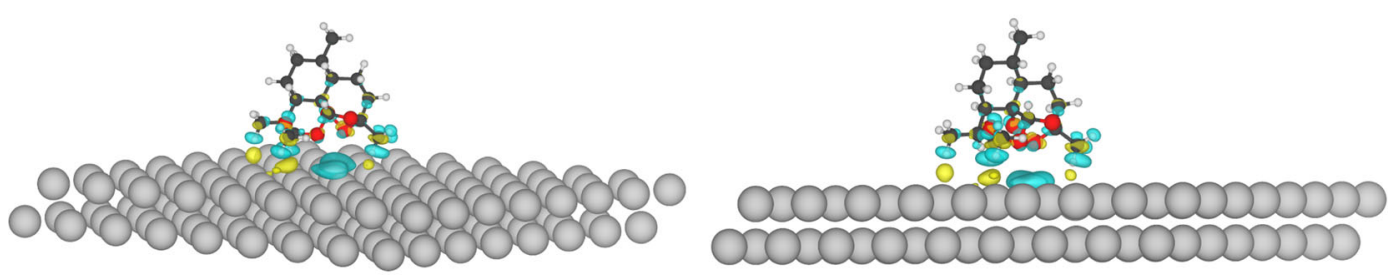

(b)
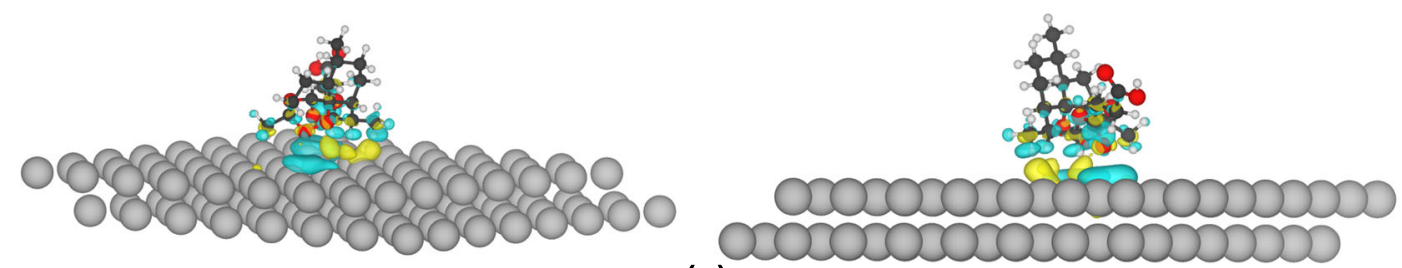

(c)

Fig. 2 The charge density difference of adsorbed Artemisinin (a), Artemether (b), and Artesunate (c) molecules on Ag(111) slab. The blue and yellow pertains to the negative and positive values corresponding to the loss and gain of electrons. The isovalue sets at 0.0005 e/a.u. ${ }^{3}$ 
Fig. 3 RMSD plots of drug molecules: Artemisinin, Artemether, and Artesunate versus simulation time. The MD trajectories after $20 \mathrm{~ns}$ have been taken for further analysis

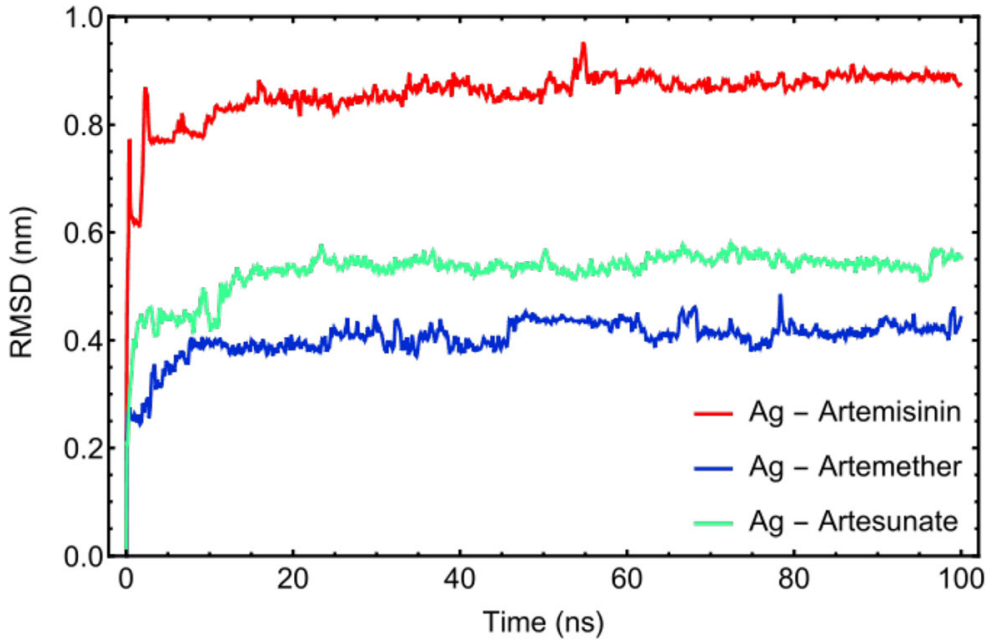

pseudopotential with scalar relativistic and non-linear core corrections for $\mathrm{Ag}$ atoms from the Quantum Espresso pseudopotential library. The energy cutoff of the planewave basis set was set to $1088 \mathrm{eV}$ and the convergence threshold of energy and force were set to $10^{-4} \mathrm{eV}$ and $10^{-3} \mathrm{eV} / \AA$. Two layers of $\mathrm{Ag}$ atoms were considered with a total number of 64 atoms per layer. During the calculations, the internal slab atoms were kept fixed at the bulk positions while the atoms in the top layer and molecule were allowed to relax. In order to avoid the interaction of slab with its replica under the periodic boundary condition, $15 \AA$ of vacuum considered in the $z$ direction (perpendicular to the slab). A gamma centred $k$-point sampling of $4 \times$ $4 \times 1$ Monkhorst-Pack grids in the first Brillouin zone of the supercell and a Gaussian smearing with a width of $\sigma=$ $0.01 \mathrm{eV}$ were used in the calculations. The convergences of the energies with respect to the energy cutoff, size of the unit cell, and $k$-point sampling were tested before the structure optimization.

The interaction of icosahedral silver clusters (the most stable particles in the range of fewer than 1130 silver atoms) with the drug molecules was simulated using molecular dynamics (MD) in a cubic box with sides of $60 \times 60 \times 60 \mathrm{~A}^{\circ}$ by GROMACS 2019 software (Abraham et al. 2019) and using the CHARMM36 force field (Huang and MacKerell Jr 2013) under the periodic boundary conditions (PBCs). Water molecules were simulated with the TIP3P model (Huang and MacKerell Jr 2013; Jorgensen et al. 1983). Silver nanocluster consisting of 147 silver atoms (diameter of $1.6 \mathrm{~nm}$ ) was fixed at the center of the simulation box while surrounded randomly with 12 molecules of each drug (Artemisinin, Artemether, or Artesunate) and water. H-bond lengths were kept constant using the LINCS routine (Hess et al. 1997) and electrostatic interactions were simulated with the particle mesh Ewald (PME) (Essmann et al. 1995) approach using the longrange cutoff of $1 \mathrm{~nm}$. The steepest descent minimization algorithm has been utilized to minimize the energy of the system for all atoms (Adcock and McCammon 2006). For equilibration, first, each system was equilibrated in an NVT ensemble (constant number of particles $(N)$, volume $(V)$, and temperature $(T))$ for $100 \mathrm{ps}$ and then in an NPT ensemble (constant number of particles $(N)$, pressure $(P)$, and temperature $(T)$ ) for 200 ps. Finally, the molecular dynamics simulation was performed for each system for 100 ns under constant conditions of $1 \mathrm{~atm}$ and $300 \mathrm{~K}$ with a time step of $2 \mathrm{fs}$. The trajectory information was analyzed using GROMACS utilities and molecular graphics and visualization were performed using VMD 1.9.3 (Humphrey et al. 1996). The force field parameters of the Artemisinin, Artemether, and Artesunate were obtained from CHARMM CGenFF (Vanommeslaeghe and MacKerell Jr 2012), and the Lennard Jones parameters for Ag nanoparticles were obtained from already published data (Sohraby et al. 2020; Kyrychenko et al. 2017).

\section{Results and discussions}

Figure 1 displays the charge distribution and the electrostatic potential map of the three drug molecules, Artemisinin, Artemether, and Artesunate. The DFT Hirschfeld point charges and electrostatic potential 
(a)
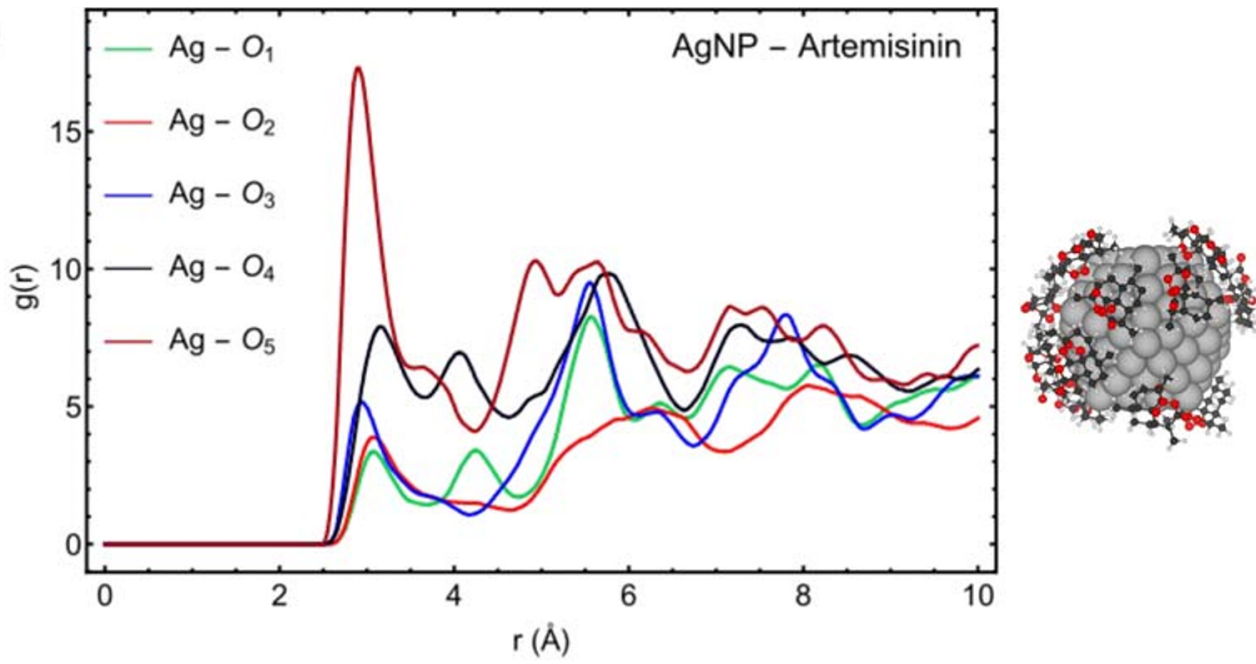

(b)
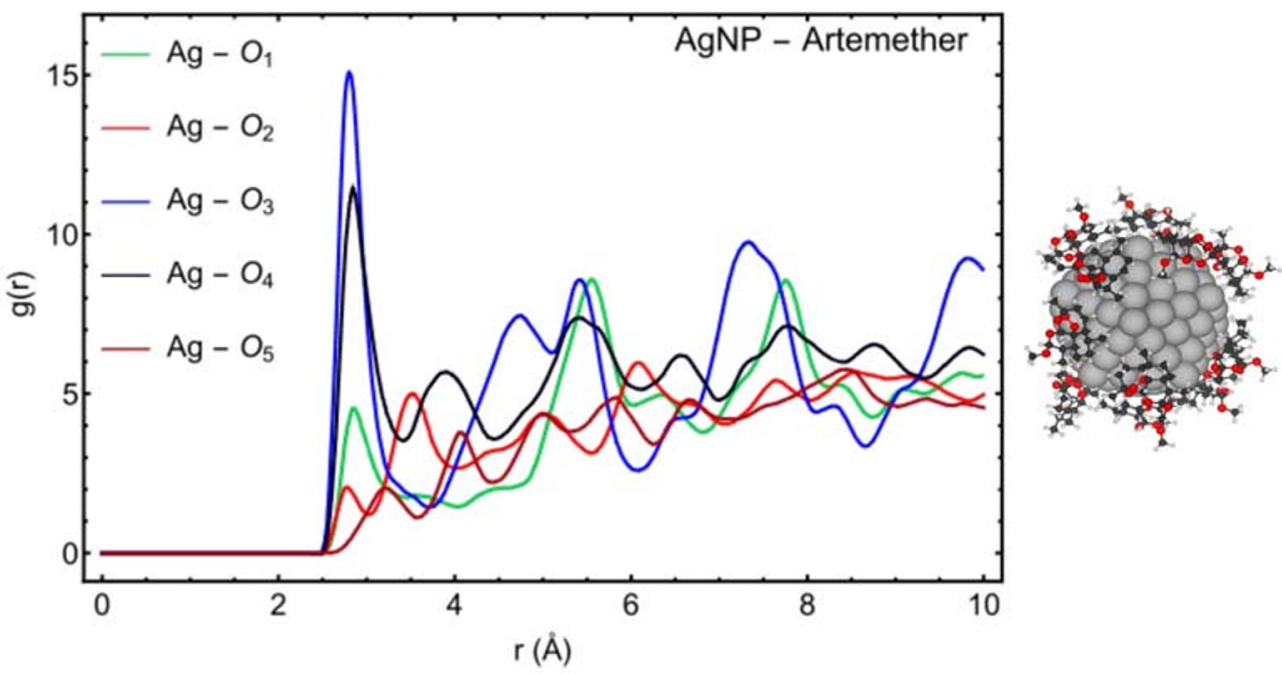

(c)

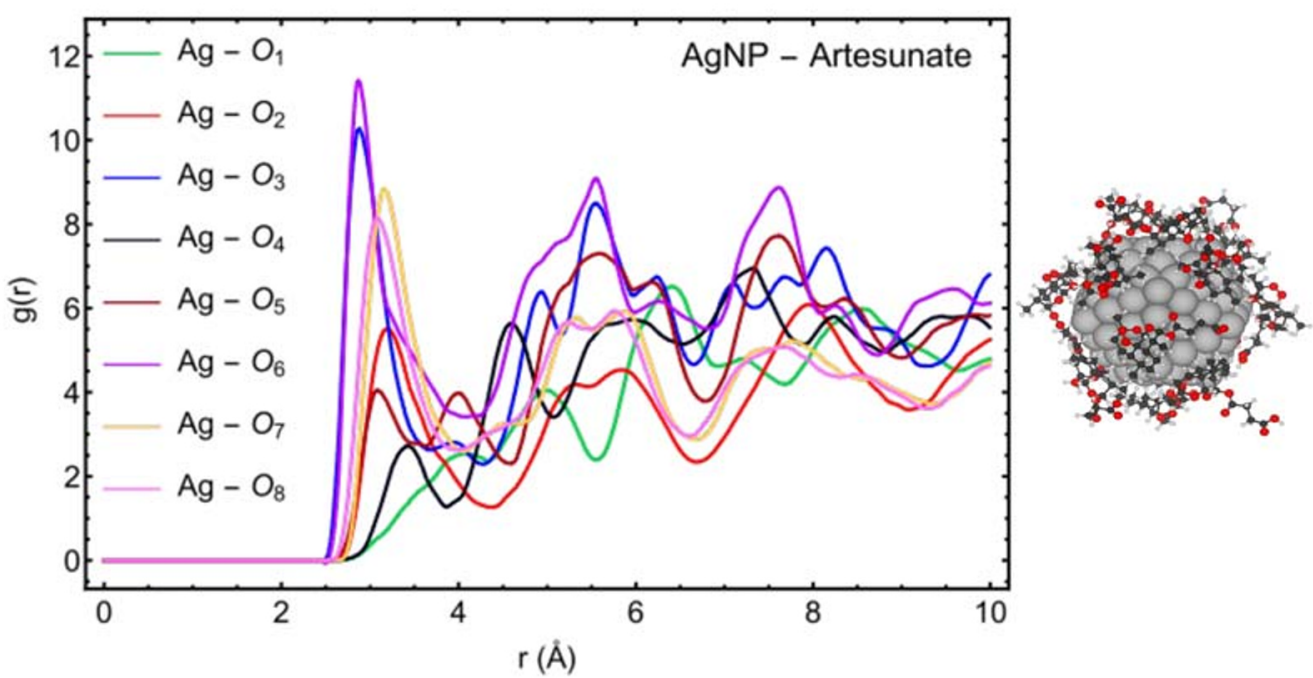

Fig. 4 RDF plots for the active sites of Artemisinin (a), Artemether (b), and Artesunate (c) with AgNP 
Fig. 5 RDF plots of Artemisinin, Artemether, and Artesunate with respect to $\mathrm{AgNP}$

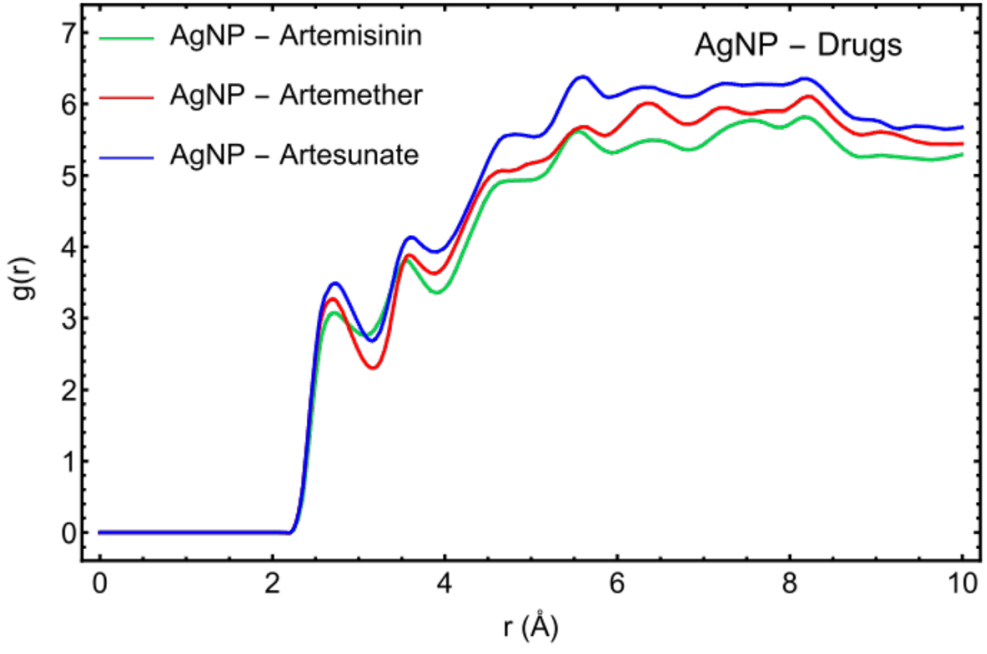

maps are obtained for the optimized molecules in water (as the solvent). The O-groups are the electron-rich site of each molecule which can donate the electron density via their lone pairs to $4 \mathrm{~d}$ and $5 \mathrm{~s}$ orbitals of the silver atom (Granatier et al. 2007; Antuek et al. 2003). Therefore, the reddish areas in the charge distribution maps in Fig. 1 demonstrate the active sites of the molecules which have the most affinity to interact with the AgNPs.

The optimized structures and charge density difference of drug molecules on $\operatorname{Ag}(111)$ surface are presented in Fig. 2. The adsorption energy for these structures were defined as $E_{\mathrm{ads}}=E_{\mathrm{Ag}-\mathrm{Art}}-E_{\mathrm{Ag}}-E_{\mathrm{Art}}$, where $E_{\mathrm{Ag}-\mathrm{Art}}$ is the total energy of the drug molecule (Artemisinin/ Artemether/Artesunate) adsorbed on the $\operatorname{Ag}(111)$ surface, while $E_{\mathrm{Ag}}$ and $E_{\mathrm{Art}}$ are the total energy of the $\mathrm{Ag}(111)$ slab and isolated drug molecule, respectively. The results for the adsorption energy are as follows: $E_{\text {ads }}^{\text {Artemisinin }}(-0.81 \mathrm{eV})$ $<E_{\text {ads }}^{\text {Artemethe }}(-0.86 \mathrm{eV})<E_{\text {ads }}^{\text {Artesunate }}(-1.07 \mathrm{eV})$. As expected from the electrostatic potential maps, the drugs interact with the Ag surface through oxygen atoms, and Artemisinin interacts weakly with the Ag surface, with interaction energy of $0.81 \mathrm{eV}$.

The charge density difference of drug molecules adsorbed on $\operatorname{Ag}(111)$ slab with a charge density isosurface value of 0.0005 e/a.u. ${ }^{3}$ is plotted in Fig. 2. This quantity accounts for the charge redistribution due to the interaction between the drug molecule and the Ag surface. The yellow color indicates an increase in the electron density after adsorbing, and the blue color indicates an electron density loss. This figure confirms the higher affinity of Artesunate to interact with $\mathrm{Ag}$ and also indicates clearly that the drug molecules interact with $\mathrm{Ag}$
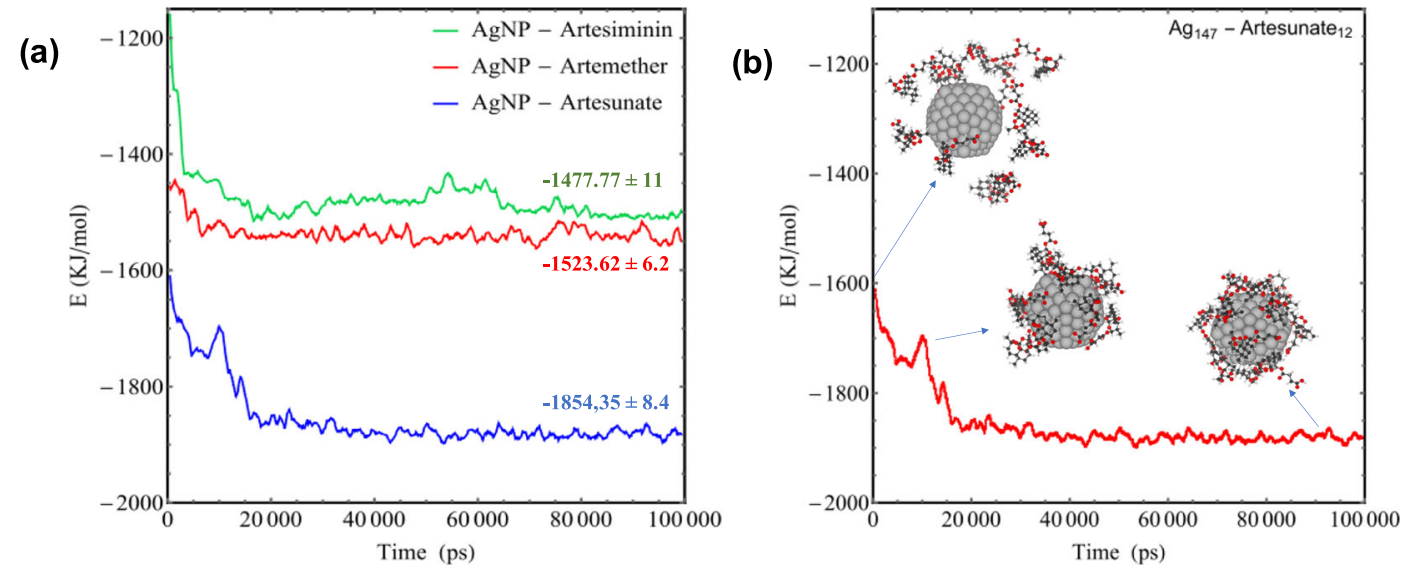

Fig. 6 a Interaction energies (vdW) between Artemisinin, Artemether, and Artesunate and AgNP. b The process of coating AgNP with 12 Artesunate molecules in terms of interaction energy 
atoms via their oxygens as discussed based on their electrostatic potential map.

Figure 3 displays the spectra of the root mean square displacement (RMSD) of Artemisinin, Artemether, and Artesunate, respectively. The plot reveals that all systems have reached their equilibrium state before $20 \mathrm{~ns}$ of simulation time, where the fluctuations of RMSDs have reduced significantly. Therefore, the MD trajectories extracted after $20 \mathrm{~ns}$ are used for further analysis.

Figure 4 describes the RDF, $g(r)$, for the active sites of Artemisinin, Artemether, and Artesunate molecules to interact with the Ag (147-atom icosahedral) nanoparticles. The radial distribution function (RDF) describes how the density of surrounding molecule varies as a function of the distance from a reference point. In this part, the arrangement of the three drug molecules and their affinities to interact with AgNP has been investigated using RDF analysis. For Artemisinin, the highest peak of atom $\mathrm{O}_{5}$ $(-0.326|e|)$ in Fig. 4a shows the highest affinity of this atom to interact with AgNP due to having more negative charges to interact with the silver nanoparticle on the surface. The atoms $\mathrm{O}_{3}(-0.201|\mathrm{e}|)$ of Artemether (Fig. 4b) and in, $\mathrm{O}_{6}(-0.296|\mathrm{e}|)$ of Artesunate (Fig. 4c) demonstrate the highest affinity to interact with AgNP. These results are in agreement with DFT calculations (see Figs. 1 and 2).

The RDF results for the drug molecules with respect to AgNP individually, Fig. 5, and the calculated vdW interaction energy, Fig. 6a, demonstrate that the Artesunate has a higher affinity to interact with AgNP compared with the Artemether and Artemisinin, which is also in agreement with the DFT calculations for the adsorption energy of drug molecules toward Ag (111) surface (see Fig. 2). The average interaction energy ( $\mathrm{vdW}$ ) based on MD simulation for Artemisinin, Artemether, and Artesunate are $-1477.77 \pm 11,-1523.62 \pm 6.2$, and $-1854.35 \pm 8.4 \mathrm{KJ} /$ mol, respectively. Moreover, as can be seen from Fig. 6 , the interaction energy fluctuations become steady and constant at the time about $17 \mathrm{~ns}$, which means that all 12 molecules coated the surface of AgNP at this time. Figure $6 \mathrm{~b}$ illustrates the coating process of AgNP with Artesunate molecules in terms of interaction energy.

\section{Conclusion}

The coating of silver nanoparticle with three common antimalarial drugs, Artemisinin, Artemether, and Artesunate, has been investigated by using the density functional theory and molecular dynamics simulations. The interaction energies of the drugs with $\mathrm{Ag}(111)$ slab clearly indicates the strength of the interaction as Artesunate $>$ Artemether $>$ Artemisinin, which is confirmed by the molecular dynamics simulation of coating 12 drug molecules on the Ag nanocluster. This work suggest that the AgNP with low toxicity and antiviral activity can be used as a drug delivery vehicle to enhance the permeability and retention (EPR) effect of these drugs against malaria and recent COVID-19 disease.

Acknowledgments The authors acknowledge the UNESCO UNISA ITHEMBA-LABS/NRF Africa Chair in Nanosciences \& Nanotechnology (U2ACN2) and the Centre for High Performance Computing (CHPC), South Africa for providing computational resources and facilities for this research project. The authors also would like to thank Dr. Zahra Jamshidi for her useful comments and discussions.

\section{Compliance with ethical standards}

Conflict of interest The authors declare that they have no conflict of interest.

\section{References}

Abid Ali Khan MM, Jain DC, Bhakuni RS, Zaim M, Thakur RS (1991) Occurrence of some antiviral sterols in Artemisia annua. Plant Sci 75(2):161-165. https://doi.org/10.1016 /0168-9452(91)90230-6

Abraham MJ, Murtola T, Schulz R, Páll S, Smith JC, Hess B, Lindahl E (2015) "GROMACS: High performance molecular simulations through multi-level parallelism from laptops to supercomputers," SoftwareX 1-2:19-25

Abraham MJ, Murtola T, Schulz R, Páll S, Smith JC, Hess B, Lindahl E (2015) GROMACS: High performance molecular simulations through multi-level parallelism from laptops to supercomputers. SoftwareX 1(2):19-25

Aderibigbe BA (2017) Metal-based nanoparticles for the treatment of infectious diseases. Molecules 22(8):1370. https://doi. org/10.3390/molecules 22081370

Alexander JW (2009) History of the medical use of silver. Surg Infect 10(3):289-292. https://doi.org/10.1089/sur.2008.9941

Aliakbari Tehrani Z, Jamshidi Z, Jebeli Javan M, Fattahi A (2012) Interactions of glutathione tripeptide with gold cluster: influence of intramolecular hydrogen bond on complexation behavior. J Phys Chem A 116(17):4338-4347. https://doi. org/10.1021/jp2080226

Antuek A, Urban M, Sadlej AJ (2003) Lone pair interactions with coinage metal atoms: weak Van der Waals complexes of the coinage metal atoms with water and ammonia. J Chem Phys 119(14):7247-7262. https://doi.org/10.1063/1.1605936 
Bazban-shotorbani S, Hasani-sadrabadi MM, Karkhaneh A, Serpooshan V (2015) Revisiting structure-property relationship of $\mathrm{pH}$-responsive polymers for drug delivery applications. J Control Release 253:46-63. https://doi.org/10.1016/j. jconrel.2017.02.021

Burdușel A-C, Gherasim O, Mihai Grumezescu A, Mogoantă L, Ficai A, Andronescu E (2018) Biomedical applications of silver nanoparticles: an up-to-date overview. Nanomaterials 8(9):681. https://doi.org/10.3390/nano8090681

Choi O, Deng KK, Kim NJ, Ross L, Surampalli RY, Hu ZQ (2008) The inhibitory effects of silver nanoparticles, silver ions, and silver chloride colloids on microbial growth. Water Res 42:3066-3074. https://doi.org/10.1016/j. watres.2008.02.021

Chowdhury NR, MacGregor-Ramiasa M, Zilm P, Majewski P, Vasilev K (2016) Chocolate silver nanoparticles: synthesis, antibacterial activity and cytotoxicity. J Colloid Interface Sci 482:151-158. https://doi.org/10.1016/j.jcis.2016.08.003

Dalrymple DG (2013) Artemisia annua, artemisinin, ACTs \& malaria control in Africa: tradition, science and public policy. Politics \& Prose Bookstore, Washington DC

Duffy PE, Mutabingwa TK (2006) Artemisinin combination therapies. Lancet 367(9528):2037-2039. https://doi.org/10.1016 /s0140-6736(06)68900-9

Efferth T (2007) Schwabe Award 2006: antiplasmodial and antitumor activity of artemisinin - from bench to bedside. Planta Med 73(4):299-309. https://doi.org/10.1055/s-2007-967138

Essmann U, Perera L, Berkowitz ML, Darden T, Lee H, Pedersen LG (1995) A smooth particle mesh Ewald method. J Chem Phys 103(19):8577-8593. https://doi.org/10.1063/1.470117

Feng QL, Wu J, Chen GQ, Cui FZ, Kim TN, Kim JO (2000) A mechanistic study of the antibacterial effect of silver ions on Escherichia coli and Staphylococcus aureus. J Biomed Mater Res 52:662-668. https://doi.org/10.1002/1097-4636 (20001215)52:4<662::aid-jbm10>3.0.co;2-3

Ferreira JF, Luthria DL, Sasaki T, Heyerick A (2010) Flavonoids from Artemisia annua L. as antioxidants and their potential synergism with artemisinin against malaria and cancer. Molecules 15(5):3135-3170. https://doi.org/10.3390 /molecules 15053135

Frisch MJ, Trucks GW, Schlegel HB et al (2009) Gaussian 09, Revision E.01, Gaussian, Inc., Wallingford CT

Galdiero S, Falanga A, Vitiello M, Cantisani M, Marra V, Galdiero M (2011) Silver nanoparticles as potential antiviral agents. Molecules 16(10):8894-8918. https://doi. org/10.3390/molecules 16108894

Geraldo DA, Needham P, Chandia N, Arratia-Perez R, Mora GC, Villagra NA (2016) Green synthesis of polysaccharidesbased gold and silver nanoparticles and their promissory biological activity. Biointerface Res. Appl. Chem 6(3): 1263-1271. http://repositorio.unab.cl/xmlui/handle/ria/1139

Giannozzi P, Baroni S, Bonini N, Calandra M et al (2009) QUANTUM ESPRESSO: a modular and open-source software project for quantum simulations of materials. J Phys Condens Matter 21(39):395502. https://doi.org/10.1088 /0953-8984/21/39/395502

Gilmore K, Osterrieder K, Seeberger PH (2020) Artemisia annua plant extracts are active against SARS-CoV-2 in vitro. In: Freie Universität Berlin. https://www.fu-berlin. $\mathrm{de} / \mathrm{en} / \mathrm{presse} / \mathrm{fup} / \mathrm{fup} / 2020 /$ fup_20_107-beifusscorona/index.html. Accessed 24 June 2020
Granatier J, Urban M, Sadlej AJ (2007) Van der Waals complexes of $\mathrm{Cu}, \mathrm{Ag}$, and $\mathrm{Au}$ with hydrogen sulfide. The bonding character. J Phys Chem A 111(50):13238-13244. https://doi.org/10.1021/jp0757098

Grimme S, Ehrlich S, Goerigk L (2011) Effect of the damping function in dispersion corrected density functional theory. J Comput Chem 32(7):1456-1465. https://doi.org/10.1002 /jcc. 21759

Henke P, Kirakci K, Kubt P, Fraiberk M, Forstov J, Mosinger J (2016) Antibacterial, antiviral, and oxygen-sensing nanoparticles prepared from electrospun materials. ACS Appl Mater Interfaces 8(38):25127-25136. https://doi.org/10.1021 /acsami.6b08234

Hess B, Bekker H, Berendsen HJ, Fraaije JG (1997) LINCS: a linear constraint solver for molecular simulations. J Comput Chem 18(12):1463-1472. https://doi.org/10.1002 /(SICI)1096-987X(199709)18:12<1463::AID-JCC4>3.0. $\mathrm{CO} ; 2-\mathrm{H}$

Huang J, MacKerell AD Jr (2013) CHARMM36 all-atom additive protein force field: validation based on comparison to NMR data. J Comput Chem 34(25):2135-2145. https://doi. org $/ 10.1002 /$ jcc. 23354

Humphrey W, Dalke A, Chulten K (1996) VMD: visual molecular dynamics. J Mol Graph 14(1):33-38. https://doi.org/10.1016 /0263-7855(96)00018-5

Ivanova N, Gugleva V, Dobreva M, Pehlivanov I, Stefanov S, Andonova V (2018) Silver nanoparticles as multi-functional drug delivery systems. https://doi.org/10.5772 /intechopen.80238

Jorgensen WL, Chandrasekhar J, Madura JD, Impey RW, Klein ML (1983) Comparison of simple potential functions for simulating liquid water. J Chem Phys 79:926-935. https://doi.org/10.1063/1.445869

Kordzadeh A, Amjad-Iranagh S, Zarif M, Modarress H (2019) Adsorption and encapsulation of the drug doxorubicin on covalent functionalized carbon nanotubes: a scrutinized study by using molecular dynamics simulation and quantum mechanics calculation. J Mol Graph Model 88:11-22. https://doi.org/10.1016/j.jmgm.2018.12.009

Kyrychenko A, Pasko DA, Kalugin ON (2017) Poly (vinyl alcohol) as a water protecting agent for silver nanoparticles: the role of polymer size and structure. Phys Chem Chem Phys 19:8742-8756. https://doi.org/10.1039/C6CP05562A

Mody VV, Siwale R, Singh A, Mody HR (2010) Introduction to metallic nanoparticles. J Pharm Bioallied Sci 2(4):282-289. https://doi.org/10.4103/0975-7406.72127

Morris D, Ansar M, Speshock J, Ivanciuc T, Qu Y, Casola A, Garofalo R (2019) Antiviral and immunomodulatory activity of silver nanoparticles in experimental RSV infection. Viruses 11(8):732. https://doi.org/10.3390/v11080732

Nedelcu I-A, Ficai A, Sonmez M, Ficai D, Oprea O, Andronescu E (2014) Silver based materials for biomedical applications. Curr Org Chem 18(2):173-184. https://doi.org/10.2174 $/ 13852728113176660141$

Pakiari AH, Jamshidi Z (2007) Interaction of amino acids with gold and silver clusters. J Phys Chem A 111(20):4391-4396. https://doi.org/10.1021/jp070306t

Patra JK, Das G, Fraceto LF, Campos EVR, Rodriguez-Torres MP, Acosta-Torres LS, Diaz-Torres LA, Grillo R, Swamy MK, Sharma S, Habtemariam S, Shin HS (2018) Nano based drug delivery systems: recent developments and future 
prospects. J Nanobiotechnol 16(71):1-33. https://doi. org/10.1186/s12951-018-0392-8

Perdew JP, Burke K, Ernzerhof M (1996) Generalized gradient approximation made simple. Phys Rev Lett 77(18):38653868. https://doi.org/10.1103/PhysRevLett.77.3865

Petrovska BB (2012) Historical review of medicinal plants' usage. Pharmacogn Rev 6(11):1-5. https://doi.org/10.4103/09737847.95849

Prusty K, Swain SK (2018) Nano silver decorated polyacrylamide/ dextran nanohydrogels hybrid composites for drug delivery applications. Mater Sci Eng C 85:130-141. https://doi. org/10.1016/j.msec.2017.11.028

Rezaee P, Akbari M, Morad R, Koochaki A, Maaza M, Jamshidi Z (2020) First principle simulation of coated hydroxychloroquine on $\mathrm{Ag}, \mathrm{Au}$ and $\mathrm{Pt}$ nanoparticle as a potential candidate for treatment of SARS-CoV-2 (COVID19), first principle simulation of coated hydroxychloroquine on $\mathrm{Ag}, \mathrm{Au}$ and $\mathrm{Pt}$ nanoparticle as a potential candidate for treatment of SARS-CoV-2 (COVID-19). In arXiv, https://arxiv.org/abs/2006.02343. Submitted on 28 May 2020

Sambasivam A, Sangwai AV, Sureshkumar R (2016) Selfassembly of nanoparticle surfactant complexes with rodlike micelles: a molecular dynamics study. Langmuir 32(5): 1214-1219. https://doi.org/10.1021/acs.langmuir.5b03689

Sohraby F, Soltanabad MH, Bagheri M, Javan MB, Moghadam MJ, Baghkheirati EK, Bagherieh Najjar MB (2020) Application of molecular dynamics in coating Agconjugated nanoparticles with potential therapeutic applications. Nano Biomed Eng 12(1):90-98. https://doi. org/10.5101/nbe.v12i1.p90-98

Stojanoski N (1999) Development of health culture in Veles and its region from the past to the end of the $20^{\text {th }}$ century. Veles Society of science and art:13-34

Suputtamongkol Y, Newton PN, Angus B, Teja-Isavadharm P, Keeratithakul D, Rasameesoraj M, Pukrittayakamee S, White NJ (2001) A comparison of oral artesunate and artemether antimalarial bioactivities in acute falciparum malaria. Br J Clin Pharmacol 52(6):655-661. https://doi.org/10.1046 j.1365-2125.2001.01458.x

Tavaf Z, Tabatabaei M, Khalafi-Nezhad A, Panahi F (2017) Evaluation of antibacterial, antibofilm and antioxidant activities of synthesized silver nanoparticles (AgNPs) and casein peptide fragments against Streptococcus mutans. Eur. J. Integr. Med 12:163-171. https://doi.org/10.1016/j. eujim.2017.05.011

U1 Haq F, Roman M, Ahmad K, Ur Rahman S, Ali Shah SM, Suleman N, Ullah S, Ahmad I, Ullah W (2020) Artemisia annua: trials are needed for COVID-19. Phytother Res 34:12. https://doi.org/10.1002/ptr.6733

Vanommeslaeghe K, MacKerell AD Jr (2012) Automation of the CHARMM general force field (CGenFF) I: bond perception and atom typing. J Chem Inf Model 52(12):3144-3154. https://doi.org/10.1021/ci300363c

Villeret B, Dieu A, Straube M, Solhonne B, Miklavc P, Hamadi S, le Borgne R, Mailleux A, Norel X, Aerts J, Diallo D, Rouzet F, Dietl P, Sallenave JM, Garcia-Verdugo I (2018) Silver nanoparticles impair retinoic acid-inducible gene I-mediated mitochondrial antiviral immunity by blocking the autophagic flux in lung epithelial cells. ACS Nano 12(2):1188-1202. https://doi.org/10.1021/acsnano.7b06934

Wang C, Xuan X, Yao W, Huang G, Jin J (2015) Anti-profibrotic effects of artesunate on bleomycin-induced pulmonary fibrosis in Sprague Dawley rats. Mol Med Rep 12(1):1291-1297. https://doi.org/10.3892/mmr.2015.3500

White NJ (2008) Qinghaosu (artemisinin): the price of success. Science 320(5874):330-334

Wong HN, Padín-Irizarry V, van der Watt ME, Reader J, Liebenberg W, Wiesner L, Smith P, Eribez K, Winzeler EA, Kyle DE, Birkholtz L-M, Coertzen D, Haynes RK (2020) Optimal 10-aminoartemisinins with potent transmission-blocking capabilities for new artemisinin combination therapies-activities against blood stage $\mathrm{P}$. falciparum including PfKI3 C580Y mutants and liver stage P. berghei parasites. Front Chem 7:901. https://doi. org/10.3389/fchem.2019.00901

Yousefpour A, Modarress H, Goharpey F, Amjad-Iranagh S (2018) Interaction of drugs amlodipine and paroxetine with the metabolizing enzyme CYP2B4: a molecular dynamics simulation study. J Mol Model 24(3):67. https://doi. org/10.1007/s00894-018-3617-8

Publisher's note Springer Nature remains neutral with regard to jurisdictional claims in published maps and institutional affiliations. 